\title{
Cognitive assessment of idiopathic intracranial hypertension patients
}

\author{
Iman A. Elbanhawy, Gihan M. Ramzy, Amal S. Ashour ${ }^{*}$ and Diana M. Khedr
}

\begin{abstract}
Background: Idiopathic intracranial hypertension $(\mathrm{IIH})$ is a disorder of unknown etiology. Goals of treatment are to preserve vision and alleviate symptoms, including intractable headache, pulsatile tinnitus, and nausea. Cognition is not addressed routinely during clinical evaluation of $\mathrm{IH}$ patients.

Objective: The current study was designed to test whether there is cognitive impairment in $\mathrm{IH}$ patients or not and to evaluate the nature and characteristics of cognitive functions in these patients.

Methods: A case-control study conducted on 40 subjects; 20 with $\mathrm{IH}$ and 20 healthy controls. Patients underwent full clinical and neurological examination as well as cognitive testing using eight psychometric tests.

Results: Patients with IIH performed significantly worse than controls in multiple cognitive domains ( $p$ value $\leq 0.05$ ). Deficits were most pronounced in working memory, executive functions, visuospatial functions, attention, and processing speed.

Conclusions: Patients with IIH have significant cognitive impairment, particularly in executive functions and memory. All domain measures showed a statistically significant difference from normal individuals, indicating that there is a form of multidomain cognitive impairment in $\mathrm{IH}$. The relationship between cognitive impairment and chronically elevated intracranial pressure and its role in contributing to patient morbidity should be considered.
\end{abstract}

Keywords: Cognition, Impairment, Morbidity, Idiopathic intracranial hypertension

\section{Introduction}

Idiopathic intracranial hypertension (IIH) is a disorder known by increased intracranial pressure (ICP) of undisclosed etiology. Chiefly, it is seen in overweight women in childbearing age [1]. Diagnosis of IIH is established by using the modified Dandy criteria [2]. There is scanty published data proving or disproving the commonly held belief that patients with IIH do not have significant cognitive impairment [3]. A recent study screened for the cognitive electrophysiological changes among IIH patients and mentioned that cognitive dysfunction in $\mathrm{IIH}$ was well estimated at neurophysiologic levels and was related to clinical assessment [4].

The hypothesis of cognitive dysfunction in patients with IIH was adopted. The current study aimed at assessing the cognition in patients with $\mathrm{IIH}$ and highlighting the pattern of cognitive impairment if present.

\footnotetext{
* Correspondence: aml_900@yahoo.com

Neurology Department, Faculty of Medicine, Cairo University, Cairo, Egypt
}

\section{Material and methods}

This case control study was conducted on two groups; IIH patients group that included $20 \mathrm{IIH}$ patients (according to modified Dandy criteria for diagnosis of IIH) [2], their age ranged from 18 to 45 years and control group which included twenty healthy volunteers matched in age, gender, and level of education to patients. All participants provided an informed consent to share in this study. The patients were recruited from Neurology outpatient clinic and Neurology department in local hospital from March 2015 to May 2016. The study was approved by two official appropriate ethical committees (intradepartmental and faculty ethical committees).

IIH patients group included patients with Mini-mental state examination (MMSE) $\geq 24$, intracranial pressure > $25 \mathrm{~cm} \mathrm{H}_{2} \mathrm{O}$ measured in lateral decubitus position, normal cerebrospinal fluid (CSF) composition, and absence of hydrocephalus or mass in brain imaging.

The current study excluded patients having major language disturbance, severe physical, visual or auditory 
impairment affecting their ability to complete testing, patients with a history of alcohol intake or any substance abuse, patients having any other medical or metabolic illness known to impair cognition, patients with current or previous major psychiatric disorder, and/or current use of drugs as anxiolytic, neuroleptic, or sedative as well as patients with known comorbidities as hypertension or diabetes mellitus.

Clinical assessment was carried for all patients including general examination of different systems assessment to exclude any associated medical illness, thorough history taking, and full neurological examination according to the IIH sheet of local Neurology Department (Additional file 1: Appendix 1).

Screening for depression was done for all participants using the Beck Depression Scale. Therefore, subjects included in the current study were those having a score of $\leq 10$ indicating minimal or no depression to abolish the effect of depression on performance in other psychometric tests (Additional file 3: Appendix 3).

Evaluation of cognitive functions using different psychometric tests was carried for all participants including Mini-Mental State Examination (MMSE) or Folstein test is used to assess subjects' orientation to time and place, instantaneous memory, short-term memory, serial subtractions or reverse spelling, constructional capacities, and use of language [5]. Modified Mini-Mental State Examination test has more standardized administration and more graded scoring than MMSE; it also assesses a broader variety of cognitive domains and covers a wider range of difficulty levels [6]. Trail making test (TMT) is used for assessment of sustained attention and information processing [7]; Passed Auditory Serial Addition Test (PASAT)) is a measure of cognitive function that specifically assesses auditory information processing speed, working memory, and flexibility, as well as calculation ability [8]; Paired Associate Learning Test (PALT) is used to assess verbal memory depending on the concept of semantic cueing [9]; and Benton Visual Retention Test (BVRT) is used to assess visual memory, visual perception, and visual constructive abilities [10] (Additional file 4).

Data was analyzed using IBM SPSS advanced statistics version 22 (SPSS Inc., Chicago, IL). Numerical data were expressed as mean and standard deviation or median and range as appropriate. Qualitative data were expressed as frequency and percentage. Chi-square test or Fisher's exact test was used to examine the relation between qualitative variables. For not normally distributed quantitative data, comparison between two groups was done using Mann-Whitney test (non-parametric $t$ test). Spearman-rho method was used to test correlation between numerical variables. All tests were two-tailed.

\section{Results}

Demographic data of the studied groups are shown in Table 1.
Most of IIH patients (13 patients) did not receive any hormonal contraception while only five patients were using oral contraceptive pills and two patients were males.

All of IIH patients (100\%) were suffering from headache with a mean duration of $16.6 \pm 4.2$ months. Clinical criteria of headache are seen in Table 2. Headache severity was assessed according to headache pain scale interpretation (Additional file 2: Appendix 2).

Eleven patients (55\%) suffered from double vision, 11 patients $(55 \%)$ suffered from transient visual obscurations (TVOS), six patients $(30 \%)$ had grade 1 papilledema, ten patients (50\%) had grade 2 papilledema, and four patients (20\%) had grade 3 papilledema. Eleven patients (55\%) suffered from tinnitus and five patients (25\%) had abducent nerve palsy.

Regarding brain imaging, ten patients $(50 \%)$ were having slit-like ventricles and empty sella in brain computerized tomography (CT) scan and one patient had superior sagittal sinus thrombosis in brain magnetic resonance venography (MRV) scan while nine patients (45\%) had normal brain imaging. Seventeen patients (85\%) were having abnormalities in perimetry in the form of enlarged blind spot and scattered scotomas while three patients (15\%) had normal perimetry.

Patients group received different lines of treatment as in Table 3.

Regarding cognitive assessment, IIH patients had statistically significant worse scores in all used psychometric tests (Table 4).

IIH patients who were not receiving hormonal contraception had statistically significant better results in PASAT 3 test $(P=0.035)$ and TMT B test $(P=0.026)$ than IIH patients who were receiving hormonal contraception.

As regards headache-associated symptoms, IIH patients who were not suffering from double vision had statistically significant better score of PASAT $3(P=0.031)$, PASAT 2 $(P=0.031)$, and TMT B $(P=0.010)$ tests than those who were suffering from it, while no significant difference was detected as regards the presence or absence of TVOs or severity of headache and papilloedema.

Furthermore, a statistically significant better score of PALT $(P=0.029)$, TMT A $(P=0.007)$, and TMT B $(P=$ $0.019)$ tests were found in those who had normal brain imaging than those who had abnormal brain imaging.

As regards effect of treatment, a statistically significant better score of BVRT $(P=0.037)$, PALT $(P=$ $0.011)$, TMT A $(P=0.037)$, and TMT B $(P=0.003)$ tests for IIH patients who were using carbonic anhydrase inhibitor than those who were not using it. Also, there was a statistically significant better score of modified mental state examination $(P=0.001)$ and TMT A $(P=0.033)$ tests for IIH patients who did shunt operation than those who did not do it. While, there was no statistically significant difference in all 
Table 1 Demographics of IIH patients and controls

\begin{tabular}{|c|c|c|c|c|}
\hline & & IIH patients $(\mathrm{No}=20)$ & Controls $(\mathrm{No}=20)$ & $P$ value \\
\hline Age $($ mean $\pm S D)$ & & $32.6 \pm 7.7$ & $30.6 \pm 7.9$ & 0.495 \\
\hline \multirow[t]{2}{*}{ Sex } & Male [No (\%)] & $2(10 \%)$ & $4(20 \%)$ & \\
\hline & Female [No (\%)] & $18(90 \%)$ & $16(80 \%)$ & 0.661 \\
\hline \multirow[t]{2}{*}{ Years of education } & < 9 years [No (\%)] & $3(15 \%)$ & $3(15 \%)$ & \\
\hline & >9 years [No (\%)] & 17 (85\%) & 17 (85\%) & 1.000 \\
\hline
\end{tabular}

IIH idiopathic intracranial hypertension, SD standard deviation, No number, \% percentage, $P$ value $\geq 0.05$ (nonsignificant)

psychometric tests for IIH patients who were taking steroids than those who were not using it.

A statistically significant positive correlation was found between IIH duration and the score of TMT A $(r=0.667$, $\mathrm{P}=0.001)$ test and TMT B $(r=0.588, P=0.006)$ test. While, there was a statistically significant negative correlation between IIH duration and the scores of MMSE $(r=-0.660$, $P=0.002)$, modified mental state examination $(r=-0.652$, $P=0.002)$, PASAT $2(r=-0.580, P=0.007)$, and PALT $(r=$ $-0.449, P=0.047)$ tests as shown in Fig. 1.

Also, a statistically significant positive correlation was detected between CSF opening pressure and both TMT A $(r=0.602, P=0.005)$ and TMT B $(r=0.513, P=0.021)$ tests, and there was a statistically significant negative correlation between it and PALT test $(r=-0.526, P=$ 0.017) as illustrated in Fig. 2 while no significant correlation was found with other tests.

Table 2 Clinical criteria of headache in $\mathrm{IH}$ patients

\begin{tabular}{llll}
\hline Criteria & & Number & Percentage (\%) \\
\hline Character & Compressing & 12 & $60 \%$ \\
& Throbbing & 4 & $20 \%$ \\
& Dull aching & 3 & $15 \%$ \\
Site & Stabbing & 1 & $5 \%$ \\
& Frontal & 9 & $45 \%$ \\
& Bitemporal & 7 & $35 \%$ \\
& Global & 2 & $10 \%$ \\
& Ocular & 1 & $5 \%$ \\
Referral & Unilateral & 1 & $5 \%$ \\
& Diffuse & 9 & $45 \%$ \\
& No referral & 7 & $35 \%$ \\
Severity & Bitemporal & 4 & $20 \%$ \\
& Mild to moderate & 9 & $45 \%$ \\
Diurnal variation & Severe & 11 & $55 \%$ \\
(morning headache) & Yes & 13 & $65 \%$ \\
Association & no & 7 & $35 \%$ \\
& Vomiting & 6 & $30 \%$ \\
& Nausea & 11 & $55 \%$ \\
& Photophobia & 16 & $80 \%$ \\
& Phonophobia & 9 & $45 \%$ \\
\hline
\end{tabular}

IIH idiopathic intracranial hypertension, \% percentage

\section{Discussion}

Cognitive function is not frequently highlighted during clinical evaluation of IIH patients. The exact pattern of cognitive impairment in those patients and its frequency is still controversial [11]. Generally, impaired function in memory, learning, visuospatial skills, learning, concentration, executive functions, and language were found $[3,12]$. Recently, cognitive affection in IIH was appreciated on neurophysiologic levels [4].

In the current study, the cognitive functions in IIH patients were examined; nature and characteristics of the dysfunction, if any, were highlighted; and finally the role of psychometric assessment in detecting cognitive impairment was evaluated. Therefore, a battery of psychometric tests was applied for assessment of global and specific cognitive functions.

Regarding clinical presentation, in the current study, all patients were presenting with headache of increase intracranial tension mainly compressing in character, frontal, diffusely referred, more on morning, increased on leaning forward, and associated with nausea, photophobia, and phonophobia. This finding coincides with the results of several studies such as [2,13-16].

Also, in the current study, $55 \%$ of the patients presented with visual problems as double vision and transient visual obscuration (TVOs). This agrees with the finding of Binder and colleagues [13] who stated that about $50 \%$ of IIH patients present with visual symptoms.

On examination, all patients in the current study had papilledema which ranged in severity from grade I to grade IV and five patients had abducent nerve palsy. This corresponds with the study of Soler and colleagues [17] who emphasized the effect of increased ICP on optic and abducent nerves.

At the start of psychometric assessment, MMSE and modified mini-mental tests were used for global screening of cognitive impairment and were found to be significantly worse in IIH patients than in control subjects. This agrees with other studies $[11,18,19]$. This was unlike Kharker and colleagues [20] results that showed normal scores among their patients. This may be due to the rough nature of this screening test (MMSE is a rapid screening test that can easily miss the cognitive impairment especially if mild. So that, the extensive cognitive 
Table 3 Lines of treatment of IIH patients

\begin{tabular}{lll}
\hline & Number & Percentage (\%) \\
\hline Carbonic anhydrase inhibitor (Acetazolamide) & 13 & $65 \%$ \\
as a monotherapy & & \\
Steroids as a monotherapy & 6 & $30 \%$ \\
Lumboperitoneal shunt & 6 & $30 \%$ \\
Optic sheath fenestration & 3 & $15 \%$ \\
\hline
\end{tabular}

$\mathrm{IIH}$ idiopathic intracranial hypertension, \% percentage

testing with the neuropsychological test battery was needed to detect cognitive impairment) in addition to small number of cases (ten cases) in their study.

On detailed assessment of specific cognitive functions in the current study, statistically significant worse results were detected in IIH patients as regards several cognitive domains including working memory (immediate and short term), information processing, visual memory, attention, calculation, psychomotor speed, and executive and visuospatial functions. Several other studies previously supported these findings as Sørensen and colleagues [11] reported difficulties in learning and memory in all their IIH patients and severely affected psychomotor speed in $20 \%$ of patients. Also, Arseni and colleagues [21] found impaired verbal memory in $24 \%$ of 85 IIH patients. Furthermore, Airaksinen and colleagues [22] found significant impairment in episodic memory and executive functions in $50 \%$ of their IIH patients.

Moreover, Kharker and colleagues [20] stated that patients with IIH had much lower scores regarding both recent and long-term memory either verbal or nonverbal than their controls, with affection of $50 \%$ of their patients. They also found impairment in executive skills in $20 \%$ of their patients and found also affection of visual spatial functions in $60 \%$ of their IIH patients. Additionally, Yri and colleagues [12] evaluated patients within 7 days of diagnosis of $\mathrm{IIH}$; they found a significant impairment in processing speed and reaction time. Lastly, Zur and colleagues [23] found marked affection in visuospatial functions of $40 \%$ of their IIH patients. Also, they found impairments of executive skills in $50 \%$ of their patients.

On the other hand $[3,12,23]$, findings showed no significant deficits in working memory. This may be due to the difference in methodology, which was mainly concerned with verbal memory than nonverbal memory, whereas in the current study both were assessed.

Regarding duration of illness, according to the current study, patients who were suffering from longstanding headache had affected executive functions, psychomotor speed, working memory, and visuospatial functions. This is in concordance with the study of Sørensen and colleagues [11] who found significant impairment in cognitive functions mainly memory in relation to duration of the disease. Also, Airaksinen and colleagues [22] found significant impairment in episodic memory and executive functions in patients diagnosed with IIH for 10 years or more when compared to the newly diagnosed ones.

On the contrary, Zur and colleagues [23] did their study on $30 \mathrm{IIH}$ patients using a NeuroTrax battery of tests for mild cognitive impairment, which used custom software installed on the testing computer. They found multidomain cognitive affection mainly attention and visuospatial processing. However, they found that there was no relation between duration of disease and cognitive function. This could be due to including patients after short period from diagnosis of headache.

In the current study, as regards severity of headache, there was no significant difference in cognitive functions between patients suffering from mild to moderate headache and those suffering from severe headache. This coincides with the studies of Yri and colleagues [12], Rist and Kurth [24], who could not elicit a correlation between cognitive dysfunction and severity of headache.

On the other hand, Moriarty and colleagues [25] and Block and Cianfrini [26] reported that severe chronic headache is associated with mild cognitive impairment in selected domains. However, it is unclear if the cognitive impairment is attributed to the pain itself or more likely mediated by coexistent depression.

Table 4 Comparison between results of psychometric tests in both groups

\begin{tabular}{llll}
\hline & IH patients (Mean \pm SD) & Controls (Mean \pm SD) & $P$ value \\
\hline Mini mental state examination (MMSE) & $28 \pm 2$ & $29.2 \pm 1$ & $0.005^{*}$ \\
Modified mental state examination & $87.2 \pm 6.5$ & $95.9 \pm 4$ & $<0.001^{*}$ \\
Passed auditory serial addition test 3 (PASAT 3) & $27.9 \pm 9.4$ & $42.4 \pm 9.3$ & $0.024^{*}$ \\
Passed auditory serial addition test 2 (PASAT 2) & $26.1 \pm 8.9$ & $38.4 \pm 7.6$ & $<0.001^{*}$ \\
Paired associate learning test (PALT) & $13.9 \pm 3.1$ & $18.7 \pm 1.6$ & $0.029^{*}$ \\
Benton visual retention test (BVRT) & $5 \pm 1.4$ & $8.3 \pm 1.4$ & $0.038^{*}$ \\
Trail making test A (TMT A)/s & $47.8 \pm 11.1$ & $34.7 \pm 5.1$ & $<0.001^{*}$ \\
Trail making test B (TMT B)/s & $106.8 \pm 12.6$ & $85.1 \pm 6.5$ & $<0.001^{*}$ \\
\hline
\end{tabular}

IIH idiopathic intracranial hypertension, SD standard deviation, MMSE mini-mental state examination, PASAT Passed Auditory Serial Addition Test, PALT Paired Associate Learning Test, TMT Trail making test, Sec second, ${ }^{*} p$ value $<0.05$ significant 

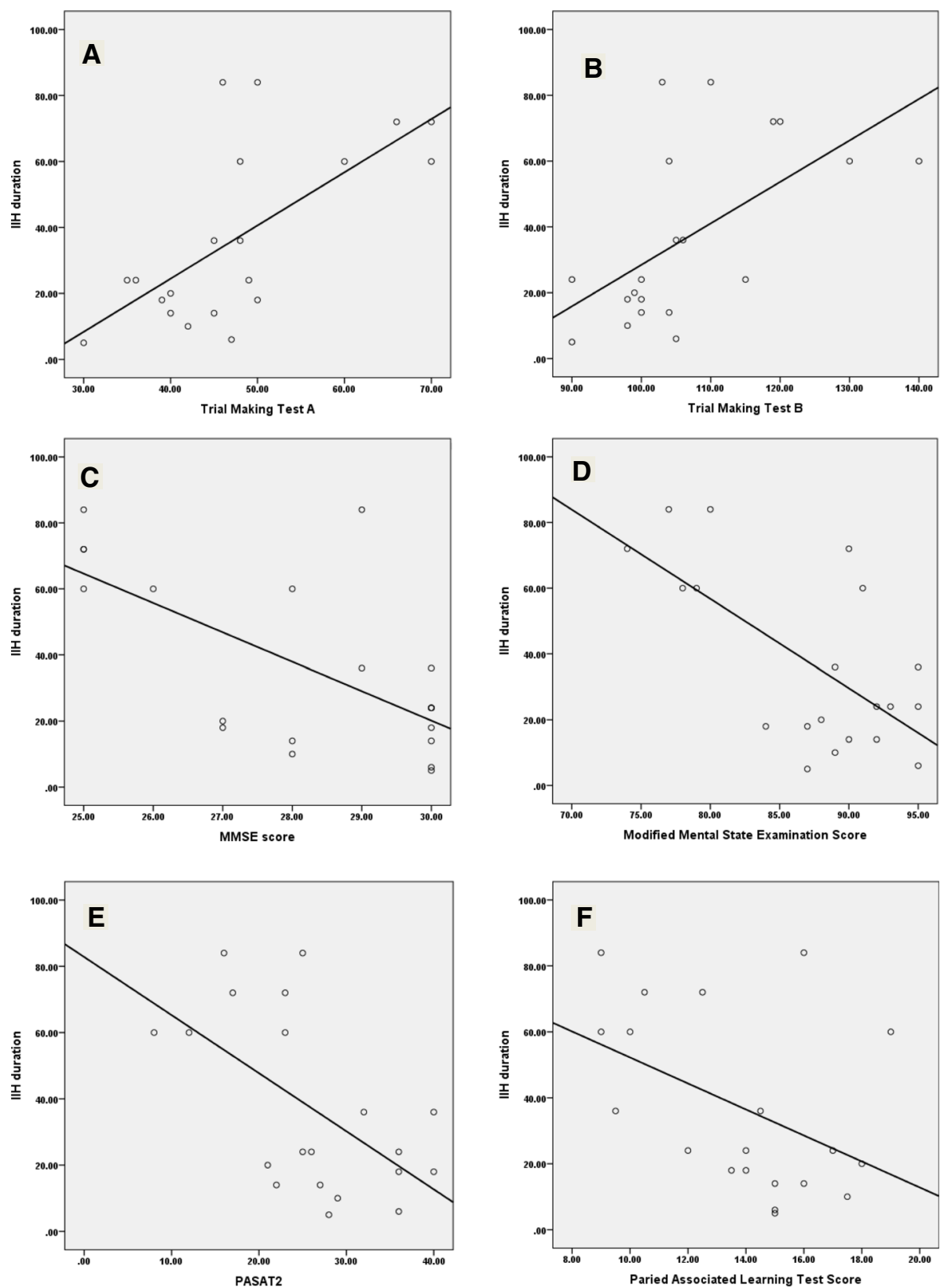

Fig. 1 Correlation between IIH duration and different psychometric tests. a Represents the correlation between IIH duration and Trail making test A (TMT A). b Represents the correlation between IIH duration and Trail making test B (TMT B). c Illustrates the correlation between IIH duration and Mini mental state examination (MMSE). $\mathbf{d}$ Illustrates the correlation between IIH duration and Modified mental state examination. e Represents the correlation between $\mathrm{IH}$ duration and Passed auditory serial addition test 2 (PASAT 2). $\mathbf{f} \|$ ustrates the correlation between IIH duration and Paired Associate Learning Test (PALT)

The CSF opening pressure, in the current study, showed a statistically significant negative correlation with attention, psychomotor speed, executive functions, working memory, and verbal fluency. This coincides with Kaplan and colleagues [3] who found that high CSF pressure markedly affect her performance in executive functions and psychomotor speed. Also, Zur and colleagues [23] stated that increase of intracranial pressure could result in deterioration in cognitive functions but did not specify which function was mostly affected.

On the contrary, Sørensen and colleagues [11] found significant impairment in cognitive functions mainly memory but not related to CSF pressure but their study was limited by small number of cases. Also, Yri and colleagues [12] evaluated patients within 7 days of diagnosis of IIH, and after 3 months of medical treatment. They 

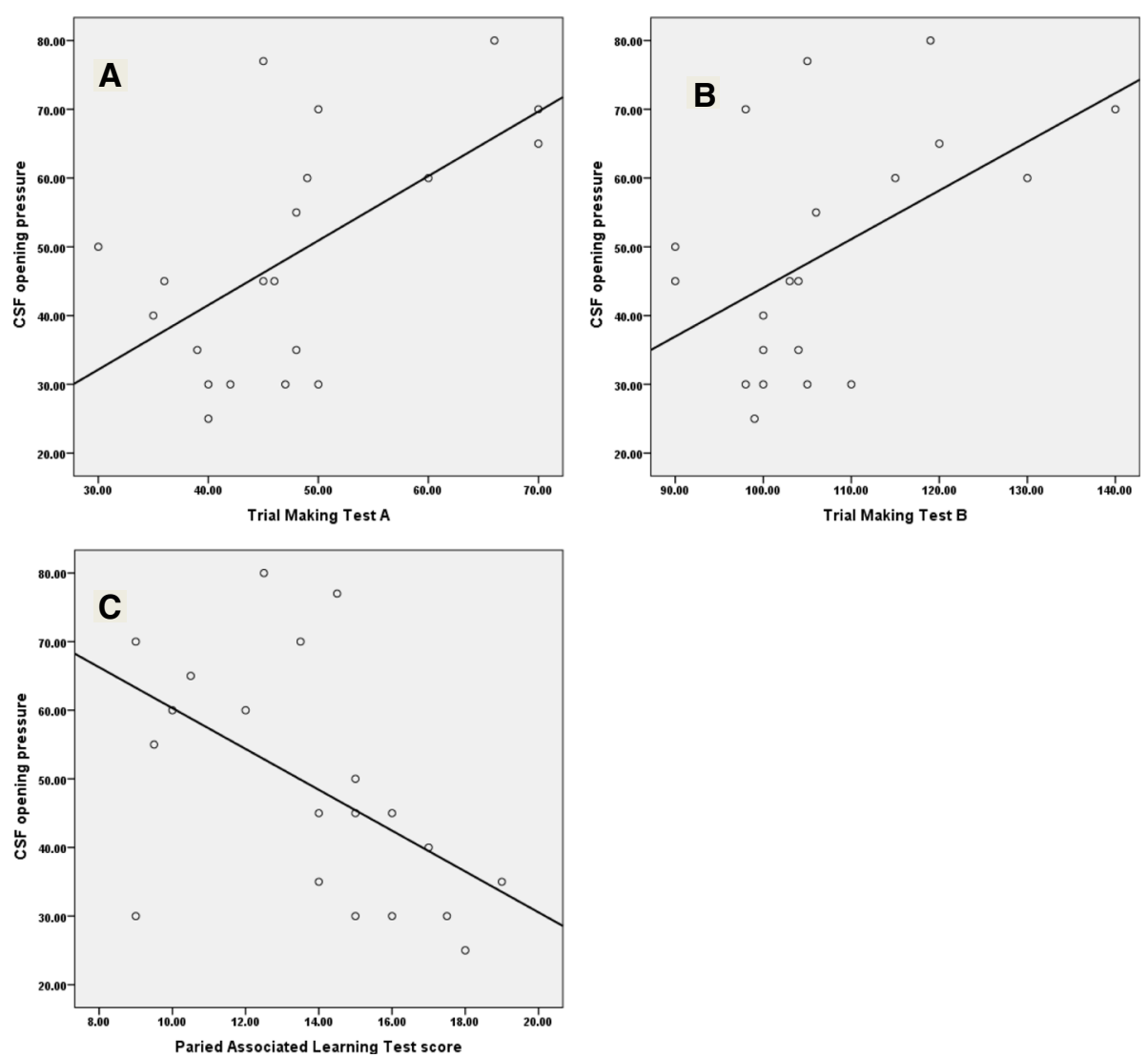

Fig. 2 Correlation between CSF opening pressure and different psychometric tests. a Represents the correlation between CSF opening pressure and Trail making test A (TMT A) b Represents the correlation between CSF opening pressure and Trail making test B (TMT B). c Represents the correlation between CSF opening pressure and Paired Associate Learning Test (PALT)

reported no significant difference in cognitive functions in relation to CSF pressure. However, inter-rater reliability for the individual data was poor.

In the current study when brain imaging results were correlated to psychometric testing, it was found that IIH patients with abnormal brain imaging (such as empty sella, slit-like ventricles, and sinus thrombosis) had worse executive functions, working memory, and psychomotor speed than those with normal ones. This in agreement with Zeitlin and Oddy [27], Bruijn and colleagues [28], and Kharker and colleagues [20] results which stated that cognitive impairment mainly in working memory and processing speed can occur in patients with confirmed cerebral venous sinus thrombosis (CVST).

In the current study, IIH patients who were using oral contraception pills showed impairment of attention, psychomotor speed, executive functions, and concentration. This goes with the study of Pletzer and colleagues [29] who found impairment in processing speed, reaction time, and memory in $25 \%$ of their patients and explained it by the possibility of structural reorganization of the brain by the effect of synthetic steroids in these pills and effect of hormones on mood increasing incidence of depression, anxiety, fatigue, neurotic symptoms, and anger.

Furthermore, IIH patients who were receiving acetazolamide achieved better scores regarding attention, psychomotor speed, working memory, and visuospatial functions than those who were not receiving it. In addition, patients who did shunt surgery had better executive functions, memory, visuospatial function, and psychomotor speed than those who did not do it.

Sørensen and colleagues [11] reported that the intellectual impairment was reversible after intensive medical or surgical measures to lower the intracranial pressure and can relieve the learning and memory disturbances even in patients with very longstanding symptoms. And Kaplan and colleagues [3] reported that cognitive impairments were reversible following medical measures to lower intracranial pressure results in decreased levels of pain, improved affect, and change in patient self-perception with resultant cessation of complains of problems in concentration and memory. Also, Kaplan and colleagues [3] found that the performance of one female patient in executive functions and psychomotor speed markedly improved after shunt insertion. Lastly, Yri and colleagues [12] 
evaluated patients within 7 days of diagnosis of IIH and after 3 months of medical treatment. They found, similar to Kaplan and colleagues [3], amultidomain impairment at first but the attention scores and visuospatial memory improved at follow-up, whereas the others stayed unchanged.

On the contrary, Zur and colleagues [23] demonstrated that cognitive decline was not related to acetazolamide treatment. Also, Yri and colleagues [12] found no convincing signs of overall cognitive improvement at the 3 months follow up.

Theories of cognitive impairment in IIH patients could involve brain dysfunction that is related to axonal flow as in optic nerve swelling or dysfunction of gray and/or white matter substance due to mechanical compression as proposed in normal pressure hydrocephalus or release of cytotoxic substances as is seen in other conditions with cognitive decline [30,31].

Cerebral blood flow $(\mathrm{CBF})$ reduction can partly explain the cognitive dysfunction in IIH patients because of the significant reduction of cerebral blood flow, particularly in those with longstanding IIH duration may lead to cerebrovascular complications [32]. There are controversial studies about CBF changes in IIH patients. Previous study by Bateman [33] found that total CBF was greater in IIH patients than that in normal control using MRI while Lorberboym and colleagues [34] observed diffuse CBF reduction in IIH patients using brain single photon emission computerized tomography (SPECT). This controversy may be due to the occurrence of the fluctuation between vascular compression and vasodilatation in $\mathrm{IIH}$ patients [35].

Proposed mechanisms of CBF changes in IIH patients are an impairment of $\mathrm{CBF}$ autoregulation, an increase of the cerebral vascular resistance, and decrease of the tissue vascular density as a result of cerebral edema [32].

Moreover, the study of the brain by event-related potential (ERP) provides a noninvasive mean of evaluating brain functioning in patients with cognitive diseases [36]. Recently, neurophysiologic tests were used to screen cognitive impairment in IIH patients by Elbanhawy and colleagues [4] who found that IIH patients had statistically significant lower amplitude and delayed latency of P300 and contingent negative variation (CNV) when compared with controls. Also, they found statistically significant negative correlation between the result of MMSE and P300 latency in IIH patients. The significant correlation between these two tests raises the possibility of similar group of brain cells are involved during their action "generators."

P300 potential is an ERP that is involved in cognitive function processing [37]. Previously, Kaipio and colleagues [38] mentioned that P300 potential has to some extent cortical generators in frontal areas. Later on, Thakur and colleagues [37] found that the P300 arises from multiple brain areas including the auditory cortex, hippocampus, and amygdala, in addition to brainstem and thalamus. Furthermore, Huang and colleagues [39] demonstrated that the electric events that explain P300 generation arise from the collaboration between frontal lobe and hippocampal/temporal-parietal function. Also, they observed that different P300 wave component variations are well correlated with frontal lobe area size and parietal area size.

Whereas the CNV is another ERP that reflects the psychological activities during increased cognitive load. It appears maximally in the electroencephalogram (EEG) over frontal and central regions [40]. Nagai and colleagues [41] observed that CNV has widespread generators involving both cortical and subcortical areas, including the primary sensorimotor area, supplementary motor area, orbitofrontal cortex (ventromedial prefrontal cortex), prefrontal area, dorsolateral and orbital prefrontal cortices, premotor and motor cortices, and temporal cortex and thalamus.

Later on, Uysal and colleagues [42] found that CNV has been well related to the activity of the prefrontal cortex and correlated with the psychological preparation activities of the person as attention, anticipation, arousal, motivation, memory, preparation, and decision making.

Previously, Yri and colleagues [12] observed that significant deficits in verbal fluency in IIH patients have been well related to frontal lobe damage.

It is possible that the lower amplitude, delayed latency of ERP (P300 and CNV), and the significant correlation between the result of MMSE and P300 latency in IIH patients reflect affection of cortical activity in the prefrontal systems. These findings implicate another possible mechanism underlying cognitive impairment in $\mathrm{IIH}$ patients.

The results of the current study showed that assessment of cognitive functions is a part of clinical evaluation of IIH patients. Also, it showed that IIH duration, use of hormonal contraception, presence of double vision, abnormal brain imaging, increase CSF opening pressure, and use of carbonic anhydrase inhibitor are different variables that affect cognitive functions in IIH patients. These variables should be taken in consideration during clinical evaluation of IIH patients for possible prevention or early detection and proper treatment of cognitive impairment if present. As the secret of improvement of cognitive dysfunction in IIH patients depend on early management and follow up of the patients to reduce CSF pressure.

\section{Limitations}

Further studies are required to detect other modifiable risk factors such as obesity and dyslipidemia on cognitive functions in IIH patients as there is common association between IIH and both obesity and dyslipidemia,. 


\section{Conclusion}

In light of the findings yielded from the current study, we can conclude that multidomain cognitive impairment is an evident common complication of IIH disease. It should be routinely investigated clinically for early detection of mild affection of cognitive functions. Thus, proper treatment planning to reduce CSF pressurewhether medical or surgical-might avoid cognitive decline, and improve it, when occurred.

\section{Additional files}

Additional file 1: Appendix 1. Neurology sheet. (DOCX $20 \mathrm{~kb}$ )

Additional file 2: Appendix 2. Headache Pain Scale Interpretation. (DOCX $19 \mathrm{~kb}$ )

Additional file 3: Appendix 3. Beck's Depression Inventory. (DOCX 24 kb)

Additional file 4: Spread sheet of psychometric tests. (XLSX $12 \mathrm{~kb}$ )

\section{Abbreviations}

BVRT: Benton Visual Retention Test; CBF: Cerebral blood flow; CNV: Contingent negative variation; CSF: Cerebrospinal fluid; CT: Computerized tomography; CVST: Cerebral venous sinus thrombosis; EEG: Electroencephalogram; ERP: Event-related potential; ICP: Increased intracranial pressure; IIH: Idiopathic intracranial hypertension; MMSE: Minimental state examination; MRV: Magnetic resonance venography; PALT: Paired Associate Learning Test; PASAT: Passed Auditory Serial Addition Test; Sec: Second; SPECT: Single photon emission computerized tomography; SSPS: Statistical Package for Social Sciences; TMT: Trail making test;

TVOS: Transient visual obscurations

\section{Acknowledgements}

Not applicable.

\section{Funding}

Not applicable.

\section{Availability of data and materials}

Spread sheet of psychometric tests is provided as Additional file 4.

\section{Authors' contributions}

IE provided the scientific background and design of study and supervised the clinical and neuropsychological part of the study. GR supervised the clinical part of the study and revised the first drafts of the manuscript. DK: history taking, clinical and neurological examination for all participants, as well as assessment of different cognitive domains. AA supervised the clinical and neuropsychological part of study, interpretation of data, and handled the final drafts of the manuscript. All authors read and approved the final manuscript.

\section{Ethics approval and consent to participate}

The current study was officially approved by an appropriate ethical committee (intradepartmental and faculty ethical committees). The approval of the ethical committee of the Faculty of Medicine, Cairo University was obtained on 16th of March 2015.

All participants of both $\mathrm{IH}$ group patients and control group gave informed consent to share in the current study.

\section{Consent for publication}

Not applicable.

\section{Competing interests}

The authors declare that they have no competing interests.

\section{Publisher's Note}

Springer Nature remains neutral with regard to jurisdictional claims in published maps and institutional affiliations.
Received: 29 July 2018 Accepted: 22 April 2019

Published online: 29 May 2019

\section{References}

1. Wall M. Idiopathic intracranial hypertension. Neurol Clin. 2010;28:593-617.

2. Friedman Dl, Jacobson DM. Diagnostic criteria for idiopathic intracranial hypertension. Neurology. 2002;59:1492-5.

3. Kaplan CP, Miner ME, Mcgregor JM. Pseudotumour cerebri: risk for cognitive impairment. Brain Inj. 1997;11:293-303.

4. Elbanhawy IA, Ramzy GM, Basheer MA, Khedr DM. Neurophysiological tests screening cognitive impairment in idiopathic intracranial hypertension patients. Egypt J Neurol Psychiat Neurosurg. 2018;54:7.

5. Folstein MF, Folstein SE, Mchugh RR. Mini mental state: a practical method for grading the cognitive state of patients for the clinician. J Psychiatr Res. 1975;12:189-98.

6. Bravo G, Herbert R. Age and education specific reference values for the min mental and modified mental state examination delivered from non demented elderly population. Int J geriat Psychiat. 1997;12:1008-18.

7. Reitan RM. The relation of the trail making test to organic brain damage. J Consult Psychol. 1955;19:393-4.

8. Gronwall DM. Paced auditory serial addition task: measure of recovery from concussion. Percept Mot Skills. 1977:44:367-73.

9. Wechsler D. A standardized memory scale for clinical use. J Psychol. 1945;19:87-95

10. Benton AL. Avisual retention test for clinical use .Archives of neurology and psychiatry, vol. 54; 1946. p. 212.

11. Sørensen PS, Thomsen AM, Gjerris F. Persistent disturbances of cognitive functions in patients with pseudotumorcerebri. Acta Neurol Scand. 1986;73: 264-8.

12. Yri HM, Fagerlund $B$, Hysse Birgitte $F$, Jensen $\mathrm{RH}$. Cognitive function in idiopathic intracranial hypertension: a prospective case-control study. BMJ Open. 2014;4(4):e004376.

13. Binder DK, Horton JC, Lawton MT, McDermott MW. Idiopathic intracranial hypertension. Neurosurgery. 2004;54:538-51.

14. Lin A, Foroozan R, Danesh-Meyer HV, De Salvo G, Savino PJ, Sergott RC. Occurrence of cerebral venous sinus thrombosis in patients with presumed idiopathic intracranial hypertension. Ophthalmology. 2006;113:2281-4.

15. Wall M. Idiopathic intracranial hypertension (pseudotumorcerebri). Curr Neurol Neurosci Rep. 2008:8:87-93.

16. González-Hernández A, Fabre-Pi O, Díaz-Nicolás S, López-Fernández JC, López-Veloso C, Jiménez-Mateos A. Headache in idiopathic intracranial hypertension. Rev Neurol. 2009:49:17-20.

17. Soler D, Cox T, Bullock P, Calver DM, Robinson RO. Diagnosis and management of benign intracranial hypertension. Arch Dis Child. 1998;78:89-94.

18. Tangalos MD. The MiniMental State Examination in general medical practice: clinical utility and acceptance. Mayo Clin Proc. 1996;71:829-37.

19. Kesler A, Mosek A, Fithlicher N, Gidron Y. Psychological correlates of idiopathic intracranial hypertension. Isr Med Assoc J. 2005;7:627-30.

20. Kharkar S, Hernandez R, Batra S, Metellus P, Hillis A, Williams MA, et al. Cognitive impairment in patients with pseudotumor cerebri syndrome. Behav Neurol. 2011;24:143-8.

21. Arseni C, Simoca I, Jipescu I, Leventi E, Grecu P, Sima A. Pseudotumorcerebri: risk factors, clinical course, prognostic criteria. Rom J Neurol Psychiatry. 1992;30:115-32.

22. Airaksinen $E$, Larsson M, Forsell $Y$. Neuropsychological functions in anxiety disorders in population-based samples: evidence of episodic memory dysfunction. J Psychiatr Res. 2005;39:207-14.

23. Zur D, Naftaliev E, Kesler A. Evidence of multidomain mild cognitive impairment in idiopathic intracranial hypertension. J Neuroophthalmol. 2015;35(1):26-30.

24. Rist PM, Kurth T. Migraine and cognitive decline: a topical review. Headache. 2013:53:589-98

25. Moriarty O, Mcguire BE, Finn DP. The effect of pain on cognitive function: a review of clinical and preclinical research. Prog Neurobiol. 2011:93:385-404.

26. Block C, Cianfrini L. Neuropsychological and neuroanatomical sequelae of chronic non-malignant pain and opioid analgesia. NeuroRehabilitation. 2013:33:343-66.

27. Zeitlin C, Oddy M. Cognitive impairment in patients with severe migraine. Br J Clin Psychol. 1984;23:27-35.

28. Bruijn S, Budde MS, Teunisse S, Haan DE, Stam J. Longterm outcome of cognition and functional health after cerebral venous sinus thrombosis. Neurology. 2000;54:1687-9. 
29. Pletzer b KM, Aichhorn M, Bergmann J, Ladumer G, Kerschbaum HH. Menstrual cycle and hormonal contraceptive use modulate human brain structure. Brain Res. 2010;1348:55-62.

30. Iddon JL, Pickard JD, Cross JJ. Specific patterns of cognitive impairment in patients with idiopathic normal pressure hydrocephalus and Alzheimer's disease: a pilot study. J Neurol Neurosurg Psychiatry. 1999;67:723-32.

31. Beeri MS, Moshier E, Schmeidler J. Serum concentration of an inflammatory glycotoxin, methylglyoxal, is associated with increased cognitive decline in elderly individuals. Mech Ageing Dev. 2011;132:583-7.

32. Bicakci K, Bicakci S, Aksungur E. Perfusion and diffusion magnetic resonance imaging in idiopathic intracranial hypertension. Acta Neurol Scand. 2006; 114(3):193-7.

33. Bateman GA. Idiopathic intracranial hypertension: priapism of the brain? Med Hypotheses. 2004;63(3):549-52.

34. Lorberboym M, Lampl Y, Kesler A, Sadeh M, Gadot N. Benign intracranial hypertension: correlation of cerebral blood flow with disease severity. Clin Neurol Neurosurg. 2001;103:33-6.

35. Levine DN. Ventricular size in pseudotumor cerebri and the theory of impaired CSF absorption. J Neurol Sci. 2000;177(2):85-94.

36. Dietrich A, Kanso R. Review of EEG, ERP, and neuroimaging studies of creativity and insight. Psychol Bull. 2010;136:822-48.

37. Thakur L, Ray K, Anand JP, Panjwani U. Event related potential (ERP) P300 after 6 months residence at 4115 meter. Indian J Med Res. 2011;134:113-7.

38. Kaipio ML, Alho K, Winkler I, Escera C, Surma-aho O, Näätänen R. Eventrelated brain potentials reveal covert distractibility in closed head injuries. Neuroreport. 1999:10(10):2125-9.

39. Huang WJ, Chen WW, Zhang X. The neurophysiology of P 300-an integrated review. Eur Rev Med Pharmacol Sci. 2015;19(8):1480-8.

40. Kochanowski J, Malara A, Broncel A. Clinical application of late negative evoked potentials. Cogtingent negative variation (CNV). Neurol Neurochir Pol. 1999;6(32):187-91.

41. Nagai Y, Critchley HD, Featherstone E, Fenwick PB, Trimble MR, Dolan RJ. Brain activity relating to the contingent negative variation: an $\mathrm{fMRl}$ investigation. Neuroimage. 2004;21(4):1232-41.

42. Uysal U, Idiman F, Idiman E, Ozakbas S, Karakas S, Bruce J. Contingent negative variation is associated with cognitive dysfunction and secondary progressive disease course in multiple sclerosis. J Clin Neurol. 2014;10(4): 296-303.

\section{Submit your manuscript to a SpringerOpen ${ }^{\circ}$ journal and benefit from:}

- Convenient online submission

- Rigorous peer review

- Open access: articles freely available online

- High visibility within the field

- Retaining the copyright to your article

Submit your next manuscript at $\boldsymbol{\nabla}$ springeropen.com 